\title{
Introduction to the minitrack on Digital and Hyperconnected Supply Chain Systems
}

\author{
Matthieu Lauras \\ IMT Mines Albi \\ matthieu.lauras@mines-albi.fr
}

\author{
Louis Faugère \\ Amazon Research and \\ Georgia Institute of Technology \\ louis.faugere@gatech.edu
}

\author{
Samuel Fosso Wamba \\ Toulouse Business School \\ s.fosso-wamba@tbs- \\ $\underline{\text { education.fr }}$
}

With the COVID-19 crisis, Supply Chain Systems (SCSs), notably in public sector, started to run in their new normality, made of systematic uncertainties, variabilities, risks but also opportunities. In parallel, the digitalization of SCSs have never been so advanced and bring some concrete perspectives for developing new approaches and paradigms to make decisions differently. In such a context, managers, public and governmental authorities have to think beyond current dogmas and practices and have to re-invent their decision support systems, particularly for SCS management. That's the core topic of this mini track.

Digital and Hyperconnected SCSs will soon be a standard, particularly in public area. SCSs have to benefit more from the new technological opportunities to better manage the uncertainties they have to cope with daily. Particularly, they have to benefit more from the hyperconnectivity potentialities that now exist in all domains, and especially in SCSs. The hyperconnectivity paradigm implies to interconnect all the stakeholders of a network on multiple levels including physics, operations, business, legal and human resources.

Such a new opportunity and problem statement open avenues for improvement and innovations for managers from both commercial and public SCSs to better manage their new normality. Notably, a smart use of Artificial Intelligence capabilities to support decision-making seem to be now a strong requirement for SCS' managers.

This year, two very interesting papers have been selected in the mini track to bring original insights regarding these issues. The first one deals with the use of reinforcement learning to support supply chain decisions while the second one suggest an innovative approach to better manage risks in supply chains.

\section{A Reinforcement Learning Powered Digital Twin to Support Supply Chain Decisions}

\section{Guillaume Martin, Raphaël Oger}

In SCSs, planning decisions are of prime importance and are probably the most difficult to make by considering the current uncertain world. Among the new approaches that have emerged during the past decade, digital twin and machine learning are probably among the most promising ones. This paper studies the possibility to build SCS digital twins from reinforcement learning algorithms. Particularly, the authors develop a methodology able to unify digital twins and reinforcement learning. They also show how to put it into practice on drug supply chain use case. Last but not least, a comparison of their approach with traditional ones is exposed to highlight the potentialities of their proposal.

\section{Supply Chain Threats and Countermeasures: From Elicitation through Optimization}

\section{Weihong (Grace) Guo, Paul Kantor, Elsayed Elsayed, Eric Rosenberg, Rong Lei, Sachin Patel, Brendan Ruskey, Fred Roberts}

Resilience in SCSs are not new topics but there is a lack of concrete solutions to evaluate and select the best countermeasures to apply to mitigate risks. This paper develops, based on simulation and optimization techniques, an original method to elicit the needed information to identify and evaluate these countermeasures automatically. Based on a bottomup / top-down approach, it also suggests how to help decision-makers to choose an optimal set of countermeasures considering a limited budget. 